\title{
Discovery of cashmere goat (Capra hircus) microRNAs in skin and hair follicles by Solexa sequencing
}

\author{
Chao Yuan ${ }^{1+}$, Xiaolong Wang ${ }^{1 \dagger}$, Rongqing Geng ${ }^{1,2+}$, Xiaolin He${ }^{1}$, Lei $\mathrm{Qu}^{3}$ and Yulin Chen ${ }^{1 *}$
}

\begin{abstract}
Background: MicroRNAs (miRNAs) are a large family of endogenous, non-coding RNAs, about 22 nucleotides long, which regulate gene expression through sequence-specific base pairing with target mRNAs. Extensive studies have shown that miRNA expression in the skin changes remarkably during distinct stages of the hair cycle in humans, mice, goats and sheep.

Results: In this study, the skin tissues were harvested from the three stages of hair follicle cycling (anagen, catagen and telogen) in a fibre-producing goat breed. In total, 63,109,004 raw reads were obtained by Solexa sequencing and 61,125,752 clean reads remained for the small RNA digitalisation analysis. This resulted in the identification of 399 conserved miRNAs; among these, 326 miRNAs were expressed in all three follicular cycling stages, whereas 3, 12 and 11 miRNAs were specifically expressed in anagen, catagen, and telogen, respectively. We also identified 172 potential novel miRNAs by Mireap, 36 miRNAs were expressed in all three cycling stages, whereas 23, 29 and 44 miRNAs were specifically expressed in anagen, catagen, and telogen, respectively. The expression level of five arbitrarily selected miRNAs was analyzed by quantitative PCR, and the results indicated that the expression patterns were consistent with the Solexa sequencing results. Gene Ontology and KEGG pathway analyses indicated that five major biological pathways (Metabolic pathways, Pathways in cancer, MAPK signalling pathway, Endocytosis and Focal adhesion) accounted for $23.08 \%$ of target genes among 278 biological functions, indicating that these pathways are likely to play significant roles during hair cycling.

Conclusions: During all hair cycle stages of cashmere goats, a large number of conserved and novel miRNAs were identified through a high-throughput sequencing approach. This study enriches the Capra hircus miRNA databases and provides a comprehensive miRNA transcriptome profile in the skin of goats during the hair follicle cycle.
\end{abstract}

Keywords: Cashmere goat, MicroRNAs, Hair cycle, Hair follicle, Skin, Solexa sequencing

\section{Background}

The mammalian hair follicle (HF) is a unique, highly regenerative neuroectodermal-mesodermal interaction system, containing a large number of stem cells [1]. The HF cycles throughout the entire life of mammals to produce new hair through stages of growth (anagen), regression (catagen) and quiescence (telogen) [2]. The HF transition between different stages is driven by a strictly controlled interaction of numerous growth stimulatory

\footnotetext{
* Correspondence: myxy11@yahoo.com.cn

${ }^{\dagger}$ Equal contributors

${ }^{1}$ College of Animal Science and Technology, Northwest A\&F University, Yangling, Shaanxi, People's Republic of China

Full list of author information is available at the end of the article
}

and inhibitory factors, which originate from the skin epithelium and mesenchyme [3]. Each stage is characterised by specific patterns of gene activation and silencing [4-6]. These conversions are controlled by the local signal environment, cytokines, hormones, neurotransmitters, as well as the transcription factors and enzymes that are recognised by key mediators in the HF cycle $[2,7]$.

MicroRNAs (miRNAs) are a large family of endogenous, non-coding RNAs, about 22-nucleotide (nt) long, which regulate gene expression through sequencespecific base pairing with target mRNAs [8]. Approximately 25,000 miRNAs have been identified in 193 species of animals, plants and microorganisms. Over the past decade, accumulating evidence has shown that

\section{Biomed Central}


miRNAs play fundamental roles in the development, function, and maintenance of tissues and cells in various organisms [9]. miRNAs are involved in the control of each stage of the hair cycle and regulate the transition between distinct hair-cycle stages by targeting different signalling pathways and transcription factors. Mice carrying a keratinocyte-specific Dicer deletion have severe alterations in $\mathrm{HF}$ morphogenesis, formation of large germ-like cysts, and hyperproliferation of the epidermis $[10,11]$. miR-203 regulates the epidermal keratinocyte differentiation and directed repression of p63 expression $[12,13]$. Moreover, miR-200b and miR-196a have been implicated in the control of HF development as potential targets for the Wnt signalling pathway [14]. The expression of miR-31 markedly increases during anagen and decreases during catagen and telogen. miR-31 is involved in the establishment of an optimal balance of gene expression in the HF, which is required for its proper growth and hair-fibre formation [15].

Cashmere goats have a double coat consisting of the over hair produced by primary HFs and the under hair (cashmere), produced by secondary HFs [16]. The growth of secondary follicles consists of three stages annually: anagen (April-November), catagen (DecemberJanuary) and telogen (February-March) [17,18].

The Shanbei White cashmere goat (SWCG), a Chinese domestic goat breed, is farmed to provide cashmere, wool and meat. Here, we present a genetic study of the miRNAs in SWCG HFs, and investigate the differential expression of miRNAs in each distinct stage of SWCG HF cycles by Solexa sequencing. We further explore their functions in the regulation of the hair growth cycle.

\section{Results}

\section{Solexa-sequencing of small RNAs}

In order to identify miRNAs involved in the three phrases (anagen, catagen and telogen) of the hair cycle, three small RNA (sRNA) libraries representing the above three phrases were constructed from a mixed pool of ten adult cashmere goat skin samples. The sRNA libraries were subsequently sequenced by Solexa sequencing. A total of $63,109,004$ raw reads were obtained. After discarding the sequences shorter than $18 \mathrm{nt}$, eliminating low-quality sequences and removing contaminants formed by adapter-adapter ligation, reads without 3 ' ligation and insert tags were obtained. Collectively, $61,125,752$ clean reads remained for further analysis (Table 1).

We then analysed the length distribution based on the three libraries and distinct sequences to assess the sequencing quality (Figure 1). Among these sequences, most were distributed in the 18-30 nt range. The highest percentages of these sRNAs were 22-nt long, which is consistent with the common size of miRNAs.

Subsequently, in order to analyse their expression and distribution in the goat genome, all of the clean Solexa reads were aligned with the goat genome sequence using SOAP software (Additional file 1: Figure S1). Of 15,997,828 reads screened in the anagen stage, 10,791,973 reads and 598,873 unique sRNAs, representing $67.46 \%$ of total reads and $45.76 \%$ of unique sRNAs, respectively, were matched by the goat genome sequence (Additional file 1: Figure S1A). To further assess the efficiency of Solexa sequencing for miRNA detection, all of the clean reads were annotated and classified using tag2annotation software (developed by Beijing Genomics Institute (BGI)), aligned against the Rfam10.1 database and the miRBase19.0 database. However, some sRNA reads may be mapped to more than one category. In order to better align every unique sRNA to one annotation, we conducted the following priority criteria: rRNA etc. (in which Genbank $>$ Rfam) $>$ conserved miRNA $>$ repeat $>$ exon $>$ intron.

The total rRNA proportion is a sign of the quality of the samples, for instance, the proportion of total rRNA should be less than $60 \%$ in plant samples [19], and $40 \%$ in animal samples (unpublished data by BGI). The total rRNA proportion in the present study was $36.5,30.47$ and $28.01 \%$ in anagen, catagen and telogen, respectively, indicating that the skin samples used were of a high quality. All of the clean reads were divided into the following

Table 1 The distribution of total small RNA tags by Solexa sequencing

\begin{tabular}{|c|c|c|c|c|c|c|c|}
\hline \multirow{2}{*}{ Type } & \multicolumn{2}{|c|}{ Anagen } & \multicolumn{2}{|c|}{ Catagen } & \multicolumn{2}{|c|}{ Telogen } & \multirow[t]{2}{*}{ Total } \\
\hline & Counts & Percent (\%) & Counts & Percent (\%) & Counts & Percent (\%) & \\
\hline total_reads & $16,861,573$ & & $18,788,688$ & & $27,458,743$ & & $63,109,004$ \\
\hline high_quality & $16,756,965$ & 100 & $18,729,213$ & 100 & $27,307,694$ & 100 & $62,793,872$ \\
\hline 3'adapter_null & 57,530 & 0.34 & 5433 & 0.03 & 44,385 & 0.16 & 107,348 \\
\hline insert_null & 103,836 & 0.62 & 81,245 & 0.43 & 76,673 & 0.28 & 261,754 \\
\hline 5'adapter_contaminants & 30,860 & 0.18 & 31,809 & 0.17 & 15,778 & 0.06 & 78,447 \\
\hline smaller_than_18nt & 566,742 & 3.38 & 498,627 & 2.66 & 154,658 & 0.57 & $1,220,027$ \\
\hline polyA & 169 & 0.00 & 187 & 0.00 & 188 & 0.00 & 544 \\
\hline clean_reads & $15,997,828$ & 95.47 & $18,111,912$ & 96.70 & $27,016,012$ & 98.93 & $61,125,752$ \\
\hline
\end{tabular}




\section{Length Distribution}

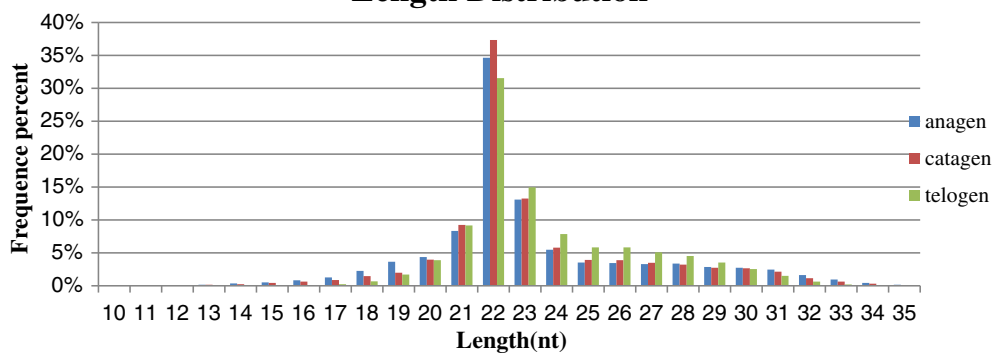

Figure 1 Sequence length distribution of the clean reads based on three total abundance and distinct sequences.

categories: exon_antisense, exon_sense, intron_antisense, intron_sense, miRNA, rRNA, repeat, scRNA, snRNA, snoRNA, srpRNA, tRNA, unan (unannotated) (Additional file 1: Figure S1A). Among them, the conserved miRNAs have $1,737,508$ total reads and 2841 unique reads, which represented $10.86 \%$ of total reads and $0.22 \%$ of unique clean reads. Of the unique reads, $56.89 \%$ were identified as potential novel miRNAs, representing $24.11 \%$ of clean reads.

\section{Expression analysis of conserved miRNAs}

Since there are no goat miRNAs available in the miRbase 19.0 database, we compared the clean reads with the miRNA precursor/mature miRNAs with known cattle sequences. Our results demonstrate that miRNA expression is abundant in the skin of cashmere goats, as a total of 399 miRNAs were found in the three stages of the HF cycle (Additional file 2: Figure S2). Among them, 326 miRNAs were expressed in all three cycling stages, whereas 3, 12 and 11 miRNAs were specifically expressed in anagen, catagen, and telogen, respectively (Figure 2).

We then analysed the differentially expressed miRNAs between the samples from every two-hair-cycle stage
(Figure 3, Additional file 3: Figure S3). Most of the expression levels were equivalent, but there were also some miRNA expression differences between the two stages (Figure 3). 68.9\% of the miRNA expression was not significant, $0.8 \%$ of the miRNAs were significantly different $(0.01 \leq \mathrm{p}<0.05)$ and $29.4 \%$ of the miRNAs were significantly different $(\mathrm{p}<0.01)$ in the catagen and anagen stages (Figure 4).

miRNAs with similar expression patterns in different sample pairs were clustered together. Clustering analysis was based on the sample difference model by using Cluster software, and the results were viewed with Java Treeview. All differentially expressed miRNAs clustered together after five rounds of clustering (Figure 5).

\section{Quantitative RT-PCR validation}

To verify the Solexa sequencing data, we randomly selected five differentially expressed miRNAs (miR-1, miR-206, miR-122, miR-222, and miR-133), and conducted quantitative RT-PCR. The relative expression levels of five selected miRNAs were consistent with the Solexa sequencing results since they had a similar trend of expression in all three periods (Figure 6).

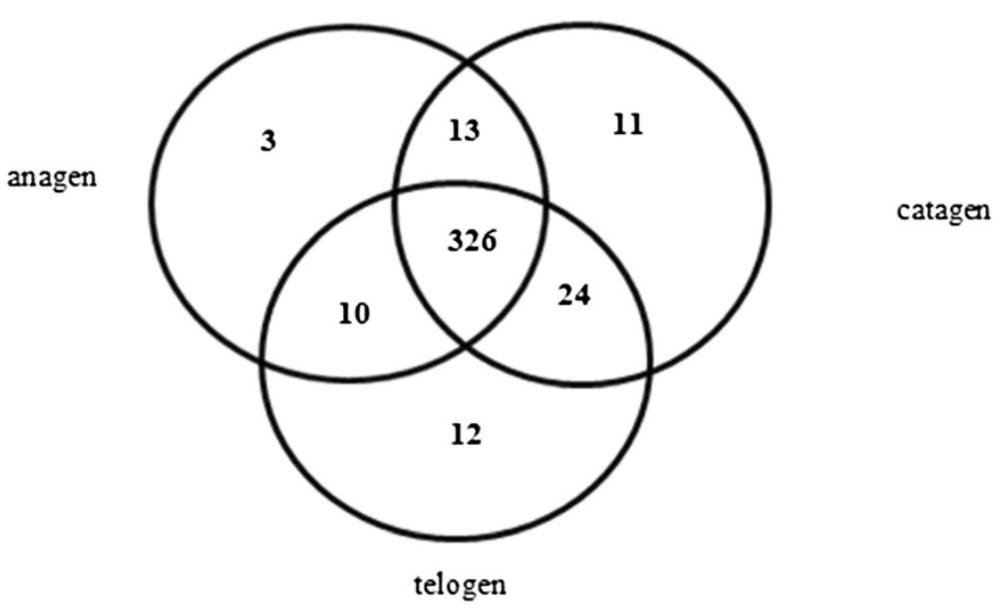

Figure 2 Venn diagram of differentially expressed conserved miRNAs at the three stages of HF cycling in cashmere goats. Numbers in parentheses are numbers of differentially expressed miRNAs at each stage. 
Figure 3 Differences of miRNA expression between the samples from every two hair cycle stage. Each point represents an miRNA. The $X$ and $Y$ axes show the expression level of miRNAs in every two samples, respectively. Red points represent miRNAs with a ratio $>2$, Blue points represent miRNAs with $1 / 2<$ ratio $<=2$, Green points represent miRNAs with ratio $<=1 / 2$, Ratio $=$ Normalised expression in Treatment/Normalised expression in Control. (A) catagen-anagen; (B) telogen-anagen; (C) telogen-catagen.

\section{Identification of novel miRNAs}

The characteristic hairpin structure of miRNA precursors can be used to predict novel miRNAs. We predicted novel miRNAs by exploring the secondary structure, the Dicer cleavage site and the minimum free energy of the unannotated small RNA reads, which could be mapped to goat genome sequences by using Mireap software. In total, 15,592,654 unannotated sequences were used to predict novel miRNAs by using the Mireap software. Among 172 potential novel miRNAs identified, 36 miRNAs were expressed in all three cycling stages, whereas 23, 29 and 44 miRNAs were specifically expressed in anagen, catagen, and telogen, respectively (Additional file 4: Figure S4, S4-1and S4-2). In addition, the length of the novel miRNA sequences ranged from 20 to $24 \mathrm{nt}$, with a distribution peak at $22 \mathrm{nt}$ and their 5 ends were comprised most frequently of uridine (U) (Additional file 4: Figure S4, S4-3).

\section{Target gene prediction for miRNAs}

miRNAs negatively regulate gene expression by base pairing between the 5 ' end of the miRNA (i.e., 2-8 nt, the "seed" region) and the $3^{\prime}$ untranslated regions (3'UTR) of target mRNAs [8,15,20-22]. Mireap software was used to predict target genes of the miRNA by searching the bovine reference gene database (http://hgdownload.cse.ucsc.edu/ goldenPath/bosTau7/bigZips/refMrna.fa.gz). In the anagen stage, 750,038 target sites in 13,860 target genes were predicted for 352 miRNAs, whereas 796,849 target sites in 13,867 target genes were predicted for 372 miRNAs in catagen, and 803,957 target sites into 13,864 target genes were predicted among 374 miRNAs in telogen.

\section{Gene Ontology (GO) enrichment and KEGG pathway analysis of target genes}

GO enrichment analysis is used for predicting candidate target genes of miRNAs. GO enrichment analysis for target genes based on the cellular component showed that 10,830 genes were termed good or better than 1 using the Component Ontology with $p$-value analysis (Additional file 5: Figure S5). More than $83.9 \%$ of genes were clustered into the cell part, followed by the intracellular part accounting for $76.2 \%$ of target genes. Analysis of molecular function showed that 10,178 genes were assigned different functions, specifically $81.6 \%$ of 


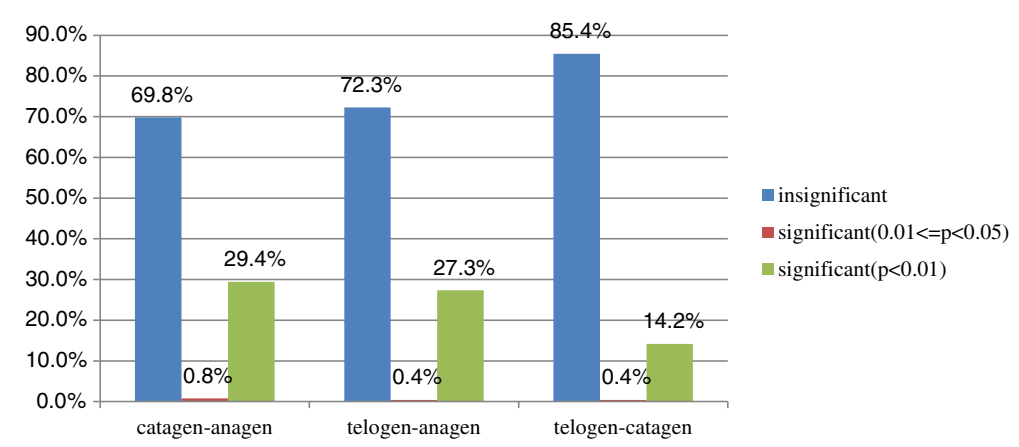

Figure 4 Changes in miRNA expression among different hair cycle stages.

genes were related to binding functions, and 9979 genes were related to biological processes. Most of the genes were involved in cellular or metabolic processes. For example, $79.4 \%$ of the genes were involved in cellular processes, and $55.8 \%$ and $47.2 \%$ of the genes were involved in metabolic processes and biological regulation, respectively.

KEGG pathway annotation showed that 10,563 target genes were annotated for 278 biological functions. Most of these genes were involved in cellular metabolism, diseases and signal transduction (Additional file 6: Figure S6). The most commonly indicated pathway was the Metabolic pathways, with 1213 genes representing $11.49 \%$ of the total target genes, followed by the Pathways in cancer (3.26\%), MAPK signalling pathway (3\%), Endocytosis (2.68\%), and Focal adhesion (2.66\%).

\section{Discussion}

The sRNA digitalisation analysis based on high-through put sequencing uses the sequencing-by-synthesis (SBS) technology predicts novel miRNAs and constructs the sRNA differential expression profile between samples from every two-hair-cycle stage, which could be used as a powerful tool for the functional studies of sRNA [23-26].

In this study, objective preliminary analysis of three cDNA libraries has shown that 22-nt sRNA is the major type of sRNA, which is consistent with the majority of sRNA-lengths in cattle [23], fish [24], goats [25,26], swine [27] and chickens [28]. Mature miRNAs, which are identical to the classical size of Dicer cleavage products [29], also have a similar trend. However, the major type of sRNA screened by Solexa sequencing in wheat is 24-nt in

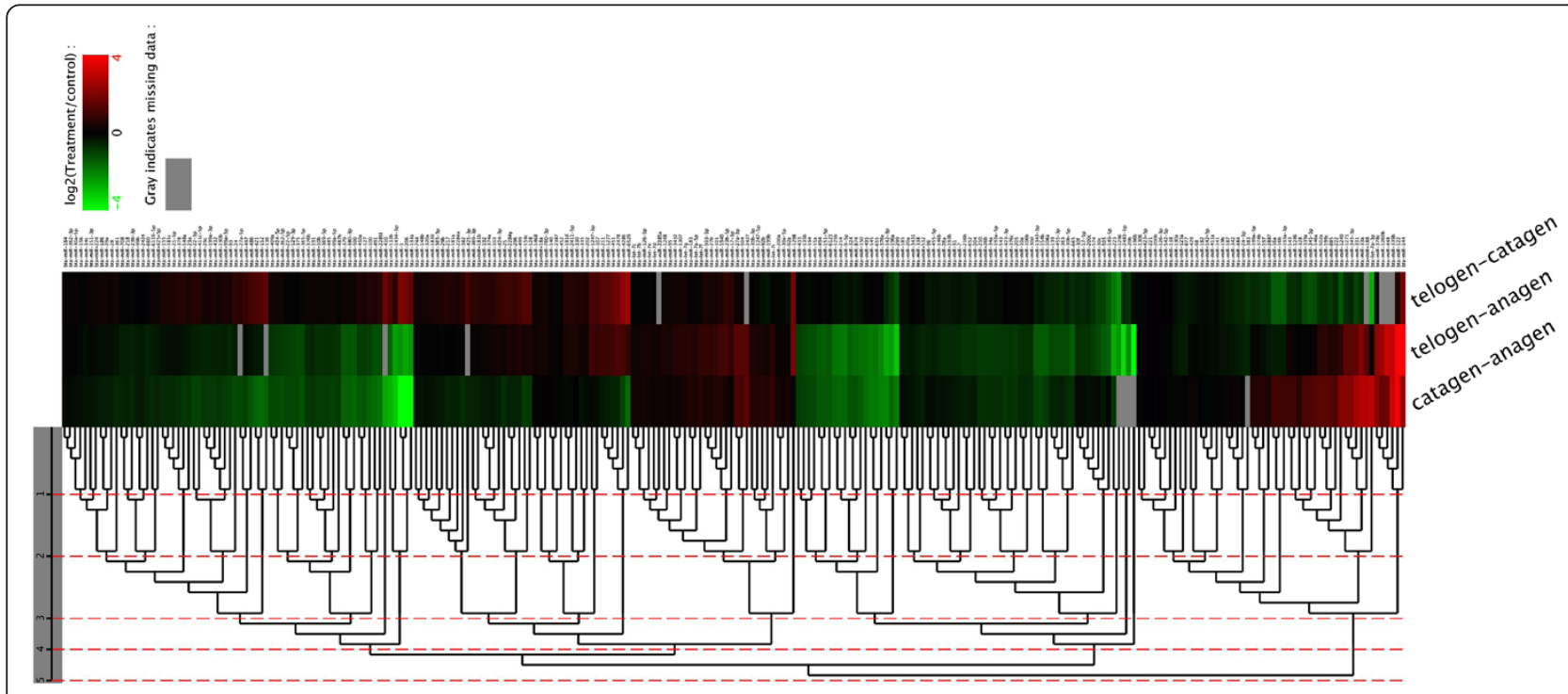

Figure 5 Clustering of miRNAs differentially expressed during HF Cycling. Red indicates that the miRNA has a higher expression level in the treatment samples; green indicates that the miRNA has a higher expression in the control samples and gray indicates that the miRNA has no expression in at least one sample. Each row in the figure represents one miRNA, and each column shows one sample pair. Each cell shows the differential expression of a miRNA in one sample pair. Heat map represents differentially expressed miRNAs between distinct stages of the hair cycle. Colour map is used to visualise the difference in expression. 


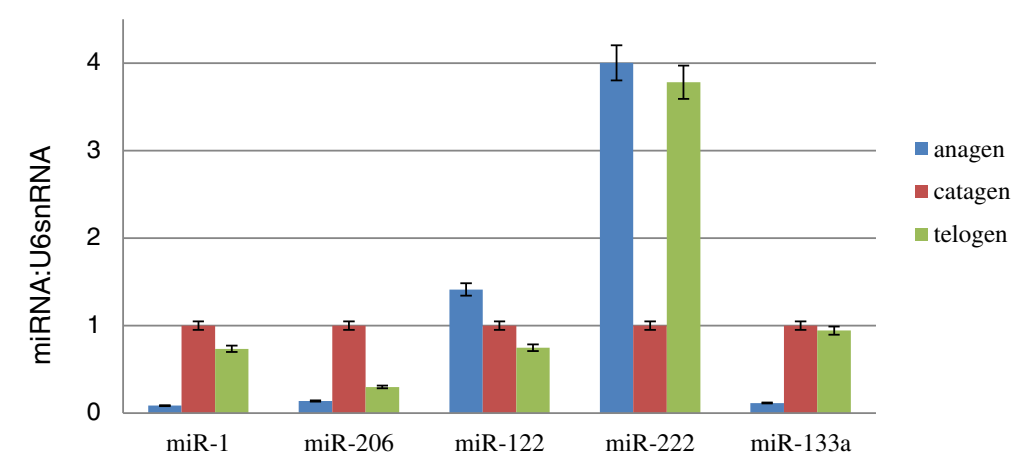

Figure 6 qPCR validation of miRNA expression in skin samples among different hair cycle stages. The abundance of miR-1, miR-206, miR-122, miR-222, and miR-133 were normalised relative to the abundance of U6 small nuclear RNA (snRNA). Error bars show the 95.0\% confidence interval of the mean.

length [30], implying that there are length differences between miRNAs from animal and plant species.

Several HF miRNAs have been shown to be involved in the regulation and forming of hair loss, hypertrichosis and skin diseases in mice and humans $[1,8]$, however, rarely have been performed in goats for fibre. Of the nine miRNAs were specifically expressed in cashmere goat dorsal skin [18], only four of them were examined in the present study: miR-1, miR-374, miR-455-3p and miR-92b. This discrepancy might be caused by inaccurate processing, base modification and sequencing/PCR errors, and analysis used inconsistencies of the database. Moreover, different genome assembly (bovine vs. caprine) may lead to the identification of various miRNAs, even in the same goat breed. For instance, compared with 352 conserved miRNAs and 83 novel miRNAs identified in the present study, Liu et al. (2012) discovered 316 conserved miRNAs and 22 novel miRNAs in another Chinese cashmere goat breed (Aerbasi White Cashmere Goat) by deep sequencing skin tissues that represented the anagen stage [26].
In the whole hair cycle, the abundance of expression of let-7a-5p, let-7f, let-7b, let-7c, let-7g, miR-199a-3p, miR-143, miR-1, and miR-320a reached their highest levels in the present study (Table 2). These miRNAs are involved in cell differentiation $[31,32]$, and proliferation [33], and the development of nerves [34], heart [35], lung [36] and muscle [37,38], suggesting that these miRNAs may play major roles in the regulation of fundamental biological processes, as well as the development of skin and HF.

HFs undergo a process of cyclical regeneration: growth, regression and quiescence. The transition from one stage to another is regulated by abundant molecules. Our results showed that the expression patterns of miR-1, miR133a, miR-133b, miR-144, miR-206, miR-299, miR-331 and miR-4286 (Additional file 3: Figure S3) were significantly different in the three stages $(\mathrm{p}<0.01)$, indicating that they may participate in the regulation of follicular transition.

According to the results of our Cluster analysis, the expression patterns of miRNAs in cashmere goat skins could

Table 2 Highly expressed miRNAs (top 10) in the three stages of HF cycling

\begin{tabular}{|c|c|c|c|c|c|}
\hline Anagen & & Catagen & & Telogen & \\
\hline miRNA & count & miRNA & count & miRNA & count \\
\hline bta-let-7a-5p & $1,975,152$ & bta-let-7a-5p & $1,661,018$ & bta-let-7a-5p & $2,270,869$ \\
\hline bta-let-7f & $1,886,542$ & bta-let-7f & $1,423,572$ & bta-let-7f & $1,885,418$ \\
\hline bta-let-7b & $1,131,783$ & bta-miR-1 & $1,273,960$ & bta-let-7b & $1,563,491$ \\
\hline bta-let-7c & 324,995 & bta-let-7b & $1,069,133$ & bta-miR-1 & 496,437 \\
\hline bta-let-7 g & 168,427 & bta-miR-199a-3p & 335,203 & bta-let-7c & 452,270 \\
\hline bta-miR-199a-3p & 159,586 & bta-let-7c & 306,476 & bta-miR-199a-3p & 391,284 \\
\hline bta-miR-143 & 155,737 & bta-let-7 g & 149,045 & bta-miR-143 & 224,149 \\
\hline bta-miR-101 & 68,706 & bta-miR-143 & 90,997 & bta-let-7 g & 185,293 \\
\hline bta-miR-1 & 58,142 & bta-miR-103 & 80,670 & bta-miR-26a & 103,535 \\
\hline bta-miR-320a & 55,595 & bta-miR-320a & 74,766 & bta-miR-320a & 87,364 \\
\hline
\end{tabular}


be divided into five types during hair cycling (Figure 5). One of the patterns is the miRNA expression level increases at anagen and then decreases during catagen and telogen (such as miR-502a, -199c, -885, -222, -1249, $1271,-345-3 p)$. In comparison with telogen and catagen, most of the miRNAs demonstrated dramatic expression changes in the anagen stage of HFs and skin, implying that these miRNAs probably participate in the formation of new hair shafts and activation of a large number of signalling pathways controlling the expression of genes encoding hair-specific molecules [2,15].

The major pathways predicted in this study were also mentioned previously. The findings of HF signal pathways in humans and mice indicates that the Wnt [39], TGF- $\beta$ [40], MAPK [41], Shh [42], Notch and JAK-STAT [43] pathways widely participate in every part of the HF cycle, development, and morphogenesis, and greatly contribute to all kinds of HF. Of the target genes identified, $3 \%$ were from the MAPK pathway, $1.52 \%$ from Wnt, $0.87 \%$ from TGF- $\beta, 0.42 \%$ from Shh, $0.43 \%$ from Notch and $1.37 \%$ from JAK-STAT. The miRNAs that correspond to these target genes will be our main candidate miRNAs for further studies on hair cycles.

\section{Conclusions}

During the anagen-catagen-telogen transformation of the hair cycle in cashmere goats, 399 conserved miRNAs and 172 novel miRNAs were found via a high-throughput sequencing approach. Our findings enrich the caprine miRNA databases and provide new insights into the miRNA transcriptome in cashmere goat skin and the HF cycle.

\section{Methods}

\section{Animal and sample preparation}

Approximately $1-\mathrm{cm}^{2}$ skin samples were harvested from the side of the body of adult goats at distinct hair cycle stages (anagen, catagen and telogen) in SWCG (five males and five females), frozen in liquid nitrogen and stored at $-80^{\circ} \mathrm{C}$ for analysis. All the experimental procedures with goats used in the present study had been given prior approval by the Experimental Animal Manage Committee of Northwest A\&F University under contract (2011-31101684).

\section{Small RNA library construction and sequencing}

Total RNA from the mixed skin tissues of ten adult goats was isolated using the RNAiso plus kit (TaKaRa, Dalian, China) according to the manufacturer's protocol. The RNA quality and quantity were determined using an Agilent 2100 Bioanalyzer (Agilent, CA, USA). Small RNA fragments of 18-30 nt in length were isolated and purified from total RNA using 15\% denaturing polyacrylamide gel electrophoresis (PAGE). Subsequently, a 3'
RNA adaptor and 5' RNA adaptor were ligated to the RNA pool using T4 RNA ligase. The sRNAs ligated with adaptors were subjected to RT-PCR amplification, and the cDNA was further amplified. The PCR products were purified using 10\% PAGE to construct an sRNA library. The sRNA libraries were constructed from skin tissue from the anagen, catagen and telogen stages, and were sequenced using an Illumina/Solexa 1G Genome Analyzer at the BGI, Shenzhen.

\section{Sequence analysis}

The basic figures from sequencing were converted into sequence data by base calling. After removing low quality reads and reads with $5^{\prime}$ primer contaminants, reads without 3' primer, reads without the insert tag, reads with poly (A), and reads shorter than $18 \mathrm{nt}$, the clean reads were obtained. We then summarised the length distribution of these clean reads. The clean reads that were obtained were compared with the ncRNAs (rRNAs, tRNAs, snRNAs, and snoRNA) deposited in the NCBI GenBank database and the Rfam 10.1 database using BLAST to annotate the sRNA sequences. The clean reads were mapped to the goat genome (http://goat.kiz. ac.cn/GGD/download.htm) by SOAP v1.11 to analyse their expression and distribution in the genome. The clean reads were aligned against the miRNA precursor/ mature miRNA of Bos taurus in miRBase19.0 (http:// www.mirbase.org/) to identify the conserved miRNAs. The unannotated sequences were used to predict potential novel miRNA candidates by Mireap (http://sourceforge. net/projects/mireap/). For an sRNA to be considered a potential novel miRNA candidate, the predicted sequences should also meet the following parameters according to Mireap: minimal miRNA sequence length (18 nt), maximal miRNA sequence length (26 nt), minimal miRNA reference sequence length (20 nt), maximal miRNA reference sequence length $(24 \mathrm{nt})$, minimal depth of Drosha/Dicer cutting site (3 nt), maximal copy number of miRNAs on reference (20 nt), maximal free energy allowed for a miRNA precursor $(-18 \mathrm{kcal} / \mathrm{mol})$, maximal space between miRNA and miRNA* (35 nt), minimal base pairs of miRNA and miRNA* (14 nt), maximal bulge of miRNA and miRNA* (4 nt), maximal asymmetry of miRNA/miRNA* duplex (5 nt), and the flank sequence length of miRNA precursor (10 nt).

The selected sequences were then folded into a secondary structure using the RNA folding program, Mfold 3.2 software. If a perfect stem-loop structure was formed, the sRNA sequence was located at one arm of the stem, and the above criteria were met, the sRNA was considered to be a potential novel miRNA candidate. We predicted the target genes of the miRNA using the Mireap software program based on the following criteria: no more than four mismatches between the sRNA and target (G-U bases 
count as 0.5 mismatches), no more than two adjacent mismatches in the miRNA/target duplex, no adjacent mismatches in positions $2-12$ of the miRNA/target duplex ( $5^{\prime}$ of miRNA), no mismatches in positions $10-11$ of the miRNA/target duplex, no more than 2.5 mismatches in positions 1-12 of the miRNA/target duplex (5' of miRNA), and the minimum free energy (MFE) of the miRNA/target duplex should be $\geq 75 \%$ of the MFE of the miRNA bound to its perfect complement.

\section{GO enrichment and KEGG pathway analyses}

We revealed the functions significantly associated with the predicted target gene candidates of the miRNAs using GO analysis. This method first maps all target gene candidates to GO terms in the database (http:// www.geneontology.org/), calculating gene numbers for each term, then uses hyper geometric testing to find significantly enriched GO terms in target gene candidates compared with the reference gene background. The calculating formula is:

$$
\mathrm{P}=1-\sum_{i=0}^{m-1} \frac{\left(\begin{array}{c}
M \\
i
\end{array}\right)\left(\begin{array}{c}
N-M \\
n-i
\end{array}\right)}{\left(\begin{array}{c}
N \\
n
\end{array}\right)}
$$

In the formula above, $\mathrm{N}$ is the number of all genes with $\mathrm{GO}$ annotation; $\mathrm{n}$ is the number of target gene candidates in N, M is the number of all genes that are annotated to a certain GO term, and $\mathrm{m}$ is the number of target gene candidates in $\mathrm{M}$. We used the Bonferroni Correction for the $p$-value to obtain a corrected $p$-value. GO terms with corrected $p$-values of $\leq 0.05$ are defined as significantly enriched in target candidate genes. This analysis is able to recognise the main biological functions for target gene candidates.

Subsequently, the main pathways in which the target candidate genes are involved were revealed by KEGG pathway analysis. The calculating formula is the same as that for $\mathrm{GO}$ analysis. Here, $\mathrm{N}$ is the number of all genes with a KEGG annotation, $\mathrm{n}$ is the number of target gene candidates in $\mathrm{N}, \mathrm{M}$ is the number of all genes annotated to a certain pathway, and $m$ is the number of target gene candidates in $\mathrm{M}$. Genes with $\mathrm{FDR} \leq 0.05$ are considered to be significantly enriched in target gene candidates. The KEGG analysis reveals the main pathways involving the target gene candidates.

\section{Differential expression analysis}

Scatter plots were used to demonstrate differentially expressed miRNA between every two follicular stages. The procedures are as follows: (1) The expression of the miRNA in two samples (control and treatment) is normalised to get the expression of transcript per million
(TPM). Normalisation formula: Normalised expression = actual miRNA count/total count of clean reads*1000000; (2) The fold-change and $p$-value are calculated from the normalised expression. Then log2ratio plot and scatter plot are generated. Fold-change formula:

Fold_change $=\log 2($ treatment $/$ control $)$

$p$-value formula:

$$
\begin{array}{ll}
\mathrm{p}(\mathrm{x} \mid \mathrm{y})=\left(\frac{\mathrm{N}_{2}}{\mathrm{~N}_{1}}\right)^{y} \frac{(\mathrm{x}+\mathrm{y}) !}{\mathrm{x} ! \mathrm{y} !\left(1+\frac{\mathrm{N}_{2}}{\mathrm{~N}_{1}}\right)^{(\mathrm{x}+\mathrm{y}+1)}} & \mathrm{D}\left(\mathrm{y} \geq \mathrm{y}_{\max } \mid \mathrm{x}\right)=\sum_{\mathrm{y}=0} \sum_{\mathrm{y} \geq \mathrm{y}_{\max }} \mathrm{p}(\mathrm{y} \mid \mathrm{x})
\end{array}
$$

After normalisation, if the miRNA gene expression amount of both samples is zero, then revise to 0.01, and if the miRNA gene expression amount for both samples is less than 1, these samples do not participate in the differential expression analysis because their expression levels are too low.

\section{Quantitative RT-PCR}

Total RNA from the mixed skin tissues of ten adult goats was isolated using the RNAiso plus kit (TaKaRa, Dalian, China), and real-time quantification of miRNAs was performed by stem-loop RT-PCR. $1 \mu \mathrm{g}$ of total RNA was reverse transcribed to CDNA using the RevertAid First Strand cDNA Synthesis Kit (Thermo Scientific Fermentas) and stem-loop RT primers (Additional file 7: Figure S7) [44]. The mix was then incubated at $42^{\circ} \mathrm{C}$ for $60 \mathrm{~min}$ and $70^{\circ} \mathrm{C}$ for $5 \mathrm{~min}$. Real-time PCR was performed using iQ5 (Bio-Rad, Hercules, CA, USA) and a standardised protocol. In a $25 \mu \mathrm{l}$ reaction mixture, $2.0 \mu \mathrm{l}$ of cDNA (at a 1:4 dilution) was used for amplification, with $12.5 \mu \mathrm{l}$ of SYBR Premix Ex Taq ${ }^{\mathrm{TM}}$ II (TaKaRa, Dalian, China), $1.0 \mu \mathrm{l}$ of specific forward primer, $1.0 \mu \mathrm{l}$ of universal primer, and $8.5 \mu \mathrm{l}$ of water. The reactions were incubated at $95^{\circ} \mathrm{C}$ for $3 \mathrm{~min}$, followed by 45 cycles of $94^{\circ} \mathrm{C}$ for $15 \mathrm{~s}, 60^{\circ} \mathrm{C}$ for $30 \mathrm{~s}$ and $72^{\circ} \mathrm{C}$ for $45 \mathrm{~s}$. The abundance of selected miRNAs was normalised relative to that of U6 snRNA. All reactions were performed in triplicate. The threshold cycle (CT) was determined using the default threshold settings and the data was analysed using the $2^{-\Delta \Delta \mathrm{Ct}}$ program.

\section{Additional files}

Additional file 1: Figure S1. The flowing results of data filtration and the distribution of sequenced small RNAs.

Additional file 2: Figure S2. Conserved miRNAs in the three stages of HF cycling.

Additional file 3: Figure S3. Differentially expressed of conserved miRNAs during HF cycling. 
Additional file 4: Figure S4. Predicted information on the novel cashmere goat miRNAs.

Additional file 5: Figure S5. GO enrichment analysis for the target genes of conserved miRNAs.

Additional file 6: Figure S6. KEGG pathways for the target genes of conserved miRNAs.

Additional file 7: Figure S7. Primers for real time $\mathrm{QPCR}$.

\section{Abbreviations}

miRNA: microRNA; HF: hair follicle; SWCG: Shanbei White cashmere goat; SRNA: small RNA; UTR: Untranslated regions; CDS: Coding sequence; GO: Gene ontology; KEEG: Kyoto encyclopedia of genes and genomes; RT-PCR: Reverse transcription PCR; BGl: Beijing Genomics Institute; MFE: Minimum free energy; TPM: Transcript per million.

\section{Competing interests}

The authors declare that they have no competing interests.

\section{Authors' contributions}

Conceived and designed the experiments: CY RG YC. Performed the experiments: CY RG. Analyzed the data: $C Y$ RG XH. Contributed reagents and materials: YC LQ. Wrote the paper: CY XW. All authors read and approved the final manuscript.

\section{Acknowledgements}

This work was supported by the Major Projects for New Varieties of Genetically Modified Organisms of China (2011ZX08008-002), the National Natural Science Foundation of China (31101684), Talent Foundation for Northwest A\&F University, and sponsored by Qing Lan Project of Jiangsu Province.

\section{Accession numbers}

The sequence data from this study have been deposited at Gene Expression Omnibus (GEO) and can be accessed with the number GSE47742.

\section{Author details}

${ }^{1}$ College of Animal Science and Technology, Northwest A\&F University, Yangling, Shaanxi, People's Republic of China. ${ }^{2}$ College of Life Science and Technology, Yancheng Teachers University, Yancheng, People's Republic of China. ${ }^{3}$ College of Life Science, Yulin University, Yulin, People's Republic of China.

\section{Received: 11 December 2012 Accepted: 24 July 2013}

Published: 28 July 2013

\section{References}

1. Krause K, Foitzik K: Biology of the Hair Follicle: The Basics. Semin Cutan Med Surg 2006, 25:2-10.

2. Stenn KS, Paus R: Controls of Hair Follicle Cycling. Physiol Rev 2001, 81:449-494.

3. Schmidt Ullrich R, Paus R: Molecular principles of hair follicle induction and morphogenesis. BioEssays 2005, 27:247-261.

4. Fuchs E: Scratching the surface of skin development. Nature 2007, 445:834-842.

5. Mikkola ML: Genetic basis of skin appendage development. Semin Cell Dev Biol 2007, 18:225-236.

6. Schneider MR, Schmidt Ullrich R, Paus R: The Hair Follicle as a Dynamic Miniorgan. Curr Biol 2009, 19:R132-R142.

7. Paus R, Foitzik K: In search of the "hair cycle clock": a guided tour. Differentiation 2004, 72:489-511.

8. Schneider MR: MicroRNAs as novel players in skin development, homeostasis and disease. British Association of Dermatologists 2012, 166:22-28.

9. Alvarez-Garcia I, Miska EA: MicroRNA functions in animal development and human disease. Development 2005, 132:4653-4662.

10. Yi R, Poy MN, Stoffel M, Fuchs E: A skin microRNA promotes differentiation by repressing 'stemness'. Nature 2008, 452:225-229.
11. Yi R, O'Carroll D, Pasolli HA, Zhang Z, Dietrich FS, Tarakhovsky A, Fuchs E: Morphogenesis in skin is governed by discrete sets of differentially expressed microRNAs. Nat Genet 2006, 38:356-362.

12. Lena AM, Shalom-Feuerstein R, Rivetti di Val Cervo P, Aberdam D, Knight RA, Melino G, Candi E: miR-203 represses 'stemness' by repressing DeltaNp63. Cell Death Differ 2008, 15:1187-1195.

13. McKenna DJ, McDade SS, Patel D, McCance DJ: MiR-203 expression in keratinocytes is dependent on regulation of p53 levels by E6. J Virol 2010, 84:10644-10652.

14. Andl T, Murchison EP, Liu F, Zhang Y, Yunta-Gonzalez M, Tobias JW, Andl CD, Seykora JT, Hannon GJ, Millar SE: The miRNA-processing enzyme dicer is essential for the morphogenesis and maintenance of hair follicles. Curr Biol 2006, 16:1041-1049.

15. Mardaryev AN, Ahmed MI, Vlahov NV, Fessing MY, Gill JH, Sharov AA Botchkareva NV: Micro-RNA-31 controls hair cycle-associated changes in gene expression programs of the skin and hair follicle. FASEB J 2010, 24:3869-3881.

16. Hai-yan Xl, Huan-min Z, Yan Z: Construction and Analysis of Subtractive cDNA Library of Secondary Follicle in Anagen in cashmere goat. Scientia Agricultura Sinica 2009, 42:3749-3754.

17. Su R, Zhang WG, Sharma R, Chang ZL, Yin J, Li JQ: Characterization of BMP2 gene expression in embryonic and adult Inner Mongolia cashmere goat (Capra hircus) hair follicles. Can J Anim Sci 2009, 89:457-462.

18. Wenguang $Z$, Jianghong $W$, Jinquan $L$, Yashizawa M: A Subset of Skin-Expressed microRNAs with Possible Roles in Goat and Sheep Hair Growth Based on Expression Profiling of Mammalian microRNAs. OMICS 2007, 11:385-396.

19. Hao D-C, Yang L, Xiao P-G, Liu M: Identification of Taxus microRNAs and their targets with high-throughput sequencing and degradome analysis. Physiol Plant 2012, 146:388-403.

20. Bartel DP: MicroRNAs: genomics, biogenesis, mechanism, and function. Cell 2004, 116:281-297.

21. Wu L, Fan J, Belasco JG: microRNAs direct rapid dead-enylation of mRNA. Proc Natl Acad Sci 2006, 103:4034-4039.

22. Easow G, Teleman AA, Cohen SM: Isolation of microRNA targets by miRNP immunopurification. RNA 2007, 13:1198-1204

23. Huang J, Ju Z, Li Q, Hou Q, Wang C, Li J, Li R, Wang L, Sun T, Hang S, Gao Y, Hou M, Zhong J: Solexa sequencing of novel and differentially expressed microRNAs in testicular and ovarian tissues in Holstein cattle. Int J Biol Sci, 7:1016-1026.

24. Chen X, Li Q, Wang J, Guo X, Jiang X, Ren Z, Weng G, Sun G, Wang X, Liu Y, Ma L, Chen JY, Wang J, Zen K, Zhang J, Zhang CY: Identification and characterization of novel amphioxus microRNAs by Solexa sequencing. Genome Biol 2009, 10:R78.

25. Ji Z, Wang G, Xie Z, Zhang C, Wang J: Identification and characterization of microRNA in the dairy goat (Capra hircus) mammary gland by Solexa Solexa-sequencing technology. Mol Biol Rep 2012, 39:9361-9371

26. Liu Z, Xiao H, Li H, Zhao Y, Lai S, Yu X, Cai T, Du C, Zhang W, Li J: Identification of conserved and novel microRNAs in cashmere goat skin by deep sequencing. PLoS One 2012, 7(12):e50001.

27. Li G, Li Y, Li X, Ning X, Li M, Yang G: MicroRNA identity and abundance in developing swine adipose tissue as determined by Solexa sequencing. J Cell Biochem 2011, 112:1318-1328.

28. Li T, Wu R, Zhang Y, Zhu D: A systematic analysis of the skeletal muscle miRNA transcriptome of chicken varieties with divergent skeletal muscle growth identifies novel miRNAs and differentially expressed miRNAs. BMC Genomics 2011, 12:186.

29. Stefani G, Slack FJ: Small non-coding RNAs in animal development. Nat Rev Mol Cell Biol 2008, 9:219-230.

30. Wei B, Cai T, Zhang R, Li A, Huo N, Li S, Gu YQ, Vogel J, Jia J, Qi Y, Mao L: Novel microRNAs uncovered by Solexa sequencing of small RNA transcriptomes in bread wheat (Triticum aestivum L.) and Brachypodium distachyon (L.). Beauv. Funct Integr Genomics 2009, 9:499-511.

31. Legesse-Miller A, Elemento O, Pfau SJ, Forman JJ, Tavazoie S, Coller HA: let-7 Overexpression leads to an increased fraction of cells in G2/M, direct down-regulation of $\mathrm{Cdc} 34$, and stabilization of Wee1 kinase in primary fibroblasts. J Biol Chem 2009, 284:6605-6609.

32. Shatseva T, Lee DY, Deng Z, Yang BB: MicroRNA miR-199a-3p regulates cell proliferation and survival by targeting caveolin-2. J Cell Sci 2011 124:2826-2836. 
33. Sun J-Y, Huang $Y$, Li J-P, Zhang $X$, Wang L, Meng Y-L, Yan B, Bian Y-Q, Zhao J, Wang W-Z, Yang A-G, Zhang R: MicroRNA-320a suppresses human colon cancer cell proliferation by directly targeting $\beta$-catenin. Biochem Biophys Res Commun 2012, 420:787-792.

34. Wulczyn FG, Smirnova L, Rybak A, Brandt C, Kwidzinski E, Ninnemann O, Strehle M, Seiler A, Schumacher S, Nitsch R: Post-transcriptional regulation of the let-7 microRNA during neural cell specification. FASEB J 2007, 21:415-426.

35. Thum T, Catalucci D, Bauersachs J: MicroRNAs: novel regulators in cardiac development and disease. Cardiovasc Res 2008, 79:562-570.

36. Harris KS, Zhang Z, McManus MT, Harfe BD, Sun X: Dicer function is essential for lung epithelium morphogenesis. Proc Natl Acad Sci USA 2006, 103:2208-2213.

37. Cordes KR, Sheehy NT, White MP, Berry EC, Morton SU, Muth AN, Lee T-H, Miano JM, Ivey KN, Srivastava D: miR-145 and miR-143 regulate smooth muscle cell fate and plasticity. Nature 2009, 460:705-710.

38. Chen J-F, Mandel EM, Thomson JM, Wu Q, Callis TE, Hammond SM, Conlon1 FL, Wang DZ: The role of microRNA-1 and microRNA-133 in skeletal muscle proliferation and differentiation. Nat Genet 2005, 38:228-233.

39. Millara SE, Willerta K, Salinasa PC, Roelinka H, Nussea R, Sussmanc DJ, Barsha GS: WNTS ignaling in the Control of Hair Growth and Structure. Dev Biol 1999, 207:133-149.

40. Foitzik K, Paus R, Doetschman T, Dotto GP: The TGF-beta2 isoform is both a required and sufficient inducer of murine hair follicle morphogenesis. Dev Biol 1999, 212:278-289.

41. Headon DJ, Overbeek PA: Involvement of a novel Tnf receptor homologue in hair follicle induction. Nat Genet 1999, 22:370-374.

42. Boras K, Hamel PA: Alx4 binding to LEF-1 regulates N-CAM promoter activity. J Biol Chem 2002, 277:1120-1127.

43. Assa-Kunik E, Torres IL, Schejter ED, Johnston DS, Shilo BZ: Drosophila follicle cells are patterned by multiple levels of Notch signaling and antagonism between the Notch and JAK/STAT pathways. Development 2007, 134:1161-1169.

44. Caifu C, Dana AR, Adam JB, Zhaohui Z, Danny HL, Julie TN, Maura B, Nan LX, Vikram RM, Mark RA, Kai QL, Kenneth JL, Karl JG: Real-time quantification of microRNAs by stem-loop RT-PCR. Nucleic Acids Res 2005, 33:e179.

doi:10.1186/1471-2164-14-511

Cite this article as: Yuan et al:: Discovery of cashmere goat (Capra hircus) microRNAs in skin and hair follicles by Solexa sequencing. BMC Genomics 2013 14:511.

\section{Submit your next manuscript to BioMed Central and take full advantage of:}

- Convenient online submission

- Thorough peer review

- No space constraints or color figure charges

- Immediate publication on acceptance

- Inclusion in PubMed, CAS, Scopus and Google Scholar

- Research which is freely available for redistribution 